\title{
Bronchoscopy Simulation in Anesthesia Resident Education
}

\author{
John McNeil, John Pawlowski
}

Beth Israel Deaconess Medical Center, Boston, USA.

Email: jsmcneil@bidmc.harvard.edu

Received March $11^{\text {th }}, 2013$; revised April $18^{\text {th }}, 2013$; accepted May $6^{\text {th }}, 2013$

Copyright (C) 2013 John McNeil, John Pawlowski. This is an open access article distributed under the Creative Commons Attribution License, which permits unrestricted use, distribution, and reproduction in any medium, provided the original work is properly cited.

\begin{abstract}
Simulation in residency training is becoming more popular but there is limited evidence showing that it can improve a resident's fund of knowledge, particularly in anesthesiology. We looked at whether a bronchoscopy simulation could improve performance on a thoracic anesthesia knowledge test administered both before and after using the simulator. Fourteen first-year anesthesiology residents completed the study with an average improvement on the test of $28 \%$ ( $<$ 0.05). We conclude that bronchoscopy simulation is an effective method of educating anesthesiology residents.
\end{abstract}

Keywords: Simulation; Bronchoscopy; Education; Residency

\section{Background}

Proficiency with the bronchoscope is essential for anesthesiology residents, but it is a difficult and demanding skill to develop. When performed in the operating room, it is often performed on unstable patients or with impatient bystanders; and most anesthesia residency curriculums do not have any dedicated time for elective bronchoscopies.

Simulation is slowly becoming a more integral part of training in the procedural specialties. Multiple studies have shown that it can lead to quantifiable improvements in procedural skill, although there are very few studies showing whether this leads to improved clinical outcomes [1]. Literature looking at anesthesiology residents and bronchoscopy is somewhat limited.

Researchers at Children's Hospital Oakland in 2002 found improved technical skills in pediatric residents after they trained on a bronchoscopy simulator, but did not look at anesthesiology residents [2]. A 2006 study reported from Germany did use anesthesia residents and found that using a simulator advanced their fiber-optic intubating skills to levels similar to that of attendings [3].

The literature isn't conclusive, however. A 2011 study at Penn State Hershey Medical Center found a wide variation in acquired skill (time to carina) in new anesthesiology residents learning nasal endoscopy on a fiber-optic simulator [4]. Also, a Canadian study in 2008 looked at respiratory therapists learning fiber-optic intubation and found no improvement when a "costly virtual reality model" was used compared with a low fidelity non-anatomic model [5].

All the literature referenced above, however, measured procedural skills. Our goal was to determine whether procedural simulation could lead to improvements in clinical knowledge of thoracic anesthesia.

\section{Procedure}

After obtaining Institutional Review Board approval, we evaluated first-year anesthesiology residents (PGY-2's) at Beth Israel Deaconess Medical Center in Boston, MA from 2009-2012 who attended a voluntary computerized bronchoscopy simulation session led by a senior cardiac/thoracic attending anesthesiologist. After giving signed consent, participants were administered a pre-simulation test of six questions related to thoracic anesthesia (doublelumen tubes, tracheal and bronchial anatomy, one-lung ventilation). Following approximately twenty-five minutes of hands-on, guided bronchoscopy simulation using an Immersion Bronchoscopy/Endoscopy Simulator (San Jose, CA), participants were administered a post-simulation test of similar difficulty and concepts. Scores were tallied anonymously and analyzed by running a paired $\mathrm{T}$ test on SPSS 15.0 (Chicago, IL).

\section{Results}

Anesthesiology residents performed better on the post- 
simulation test than the pre-simulation test (62\% correct vs. $34 \%$ correct respectively, a difference of $28 \%$ ). These results were statistically significant with a p-value of 0.012 .

\begin{tabular}{|c|c|c|c|}
\hline Resident & $\begin{array}{l}\text { Pre-simulation } \\
\% \text { correct }\end{array}$ & $\begin{array}{l}\text { Post-simulation } \\
\% \text { correct }\end{array}$ & Difference \\
\hline 1 & 40 & 100 & 60 \\
\hline 2 & 60 & 80 & 20 \\
\hline 3 & 40 & 0 & -40 \\
\hline 4 & 20 & 80 & 60 \\
\hline 5 & 40 & 60 & 20 \\
\hline 6 & 0 & 40 & 40 \\
\hline 7 & 20 & 60 & 40 \\
\hline 8 & 40 & 60 & 20 \\
\hline 9 & 20 & 60 & 40 \\
\hline 10 & 60 & 80 & 20 \\
\hline 11 & 20 & 60 & 40 \\
\hline 12 & 20 & 20 & 0 \\
\hline 13 & 40 & 80 & 40 \\
\hline 14 & 60 & 20 & -40 \\
\hline & & \multicolumn{2}{|c|}{ Difference } \\
\hline & Z & \multicolumn{2}{|c|}{$-2.516(\mathrm{a})$} \\
\hline \multicolumn{2}{|c|}{ Asymp. Sig. (2-tailed) } & \multicolumn{2}{|c|}{0.012} \\
\hline
\end{tabular}

\section{Discussion}

In order to correctly position a double lumen tube or bronchial blocker, anesthesia residents need to know how to manipulate a fiberoptic bronchoscope, how to recognize the normal anatomy and how to deduce when the tube placement or blocker placement is abnormal. Virtual reality bronchoscopy allows residents to explore the airway bronchial anatomy in a safe setting, without clinical concerns or time pressures [6]. In addition, instruction can be provided regarding the gentle manipulation of the bronchoscope in order to minimize damage of the instrument. The virtual bronchoscopy programs also allow simulation of coughing, hemorrhage and the presence of endobronchial tumors on demand. Therefore, the virtual bronchoscopy session provides guaranteed exposure of the trainee to common problems and predictable pathologies.

In this study, the right- and left-sided bronchial anatomy was illustrated and compared, to allow the resident to confirm the correct placement of a double lumen tube or a bronchial blocker. All residents were asked to place the bronchoscope into the right upper lobe, the bronchus intermedius, the left main bronchus and the left lower lobe. During this portion of the virtual bronchoscopic examination, common locations of malpositioned tubes were mentioned. In a subsequent model, residents placed double lumen tubes and bronchial blockers to achieve lung isolation. Correct position of the tube and correct position of the inflated balloons was reinforced. By requiring the demonstration of correct positioning of the bronchial airway device to achieve lung isolation, the instructor confirmed not only ability to identify airway anatomy but also to correctly position and employ the devise under fiberoptic guidance.

To document the acquisition of factual knowledge about bronchial anatomy and lung isolation techniques a series of pre- and post-tests were developed. These tests were written by investigators other than the Principal Investigator (JP) and were administered and graded by investigators other than the PI. This was done to avoid the conflict of the PI, who was also the division director and the person in charge of the resident's thoracic anesthesia rotation from having direct knowledge of any individual resident's performance on the pre- and post-tests. As a result, the pre- and post-tests were initially found to be of unequal difficulty and this was discovered on annual review. As a result, the post-test was rewritten for the subsequent residents. All data presented does not include the results from the first year of investigation.

The findings do show a significant improvement in cognitive information as evidenced by improved scores on a multiple choice examination immediately following an instructional exercise using virtual bronchoscopy and lung isolation simulation. These residents were first year anesthesia residents and had little prior practical experience with either fiberoptic bronchoscopy or lung isolation techniques. These residents had also not rotated on any thoracic anesthesia rotations prior to the simulation exercise. Previous surveys had indicated both a lack of experience and a lack of confidence toward bronchoscopic examination.

A limitation of this study is that no correlation was made as to subsequent bronchoscopic experiences of the residents in real patients. Also, there was no evaluation of injuries and complications in patients or any breakages of bronchoscopic equipment in procedures performed by residents who had undergone this simulation training. It is hoped that subsequent studies might attempt to find a reduction in patient injuries and equipment damages when residents receive extensive simulation training prior to their clinical rotations. Lastly, although deemed equivalent by the Principal Investigator, there is a possibility that the pre-test was easier than the post-test and improvements in performance would have resulted without any simulation work. Future studies will randomize participants to receive one of two tests as a pre-test and the other as a post-test to eliminate this possibility. 


\section{Conclusion}

Bronchoscopy simulation is an effective method of educating anesthesiology residents.

\section{REFERENCES}

[1] Y. Okuya, E. O. Bryson, S. DeMaria Jr., et al., “The Utility of Simulation in Medical Education: What Is the Evidence? Mount Sinai Journal of Medicine, Vol. 76, No. 4, 2009, pp. 330-343. doi:10.1002/msj.20127

[2] R. Rowe and R. A. Cohen, "An Evaluation of a Virtual Reality Airway Simulator,” Anesthesia \& Analgesia, Vol. 95, No. 1, 2002, pp. 62-66.

doi:10.1097/00000539-200207000-00011

[3] K. Goldmann and T. Steinfeldt, “Acquisition of Basic Fiberoptic Intubation Skills with a Virtual Reality Airway
Simulator,” Journal of Clinical Anesthesia, Vol. 18, No. 3, 2006, pp. 173- 178. doi:10.1016/j.jclinane.2005.08.021

[4] P. G. Dalal, G. B. Dalal, L. Pott, et al., "Learning Curves of Novice Anesthesiology Residents Performing Simulated Fibreoptic Upper Airway Endoscopy,” Canadian Journal of Anesthesia, Vol. 58, No. 9, 2011, pp. 802-809. doi:10.1007/s12630-011-9542-2

[5] D. B. Chandra, G. L. Savoldelli, H. S. Joo, et al., "Fiberoptic Oral Intubation: The Effect of Model Fidelity on Training for Transfer to Patient Care,” Anesthesiology, Vol. 109, No. 6, 2008, pp. 1007-1013. doi:10.1097/ALN.0b013e31818d6c3c

[6] J. Neelankavil, et al., "Difficult Double-Lumen Tube Placement Complicated by an Intraoperative Pneumothorax," Simulation in Healthcare, Vol. 7, No. 2, 2012, pp. 127 132. doi:10.1097/SIH.0b013e3182232ec0 